\title{
MYRINGOPLASTY OF CENTRAL TYMPANIC MEMBRANE PERFORATION WITH A FAT GRAFT FROM THE EAR LOBULE AND PLATELET RICH PLASMA.
}

\author{
Ahmed Roshdy Ebrahim M.B.B. Ch. ${ }^{c}$, Yasser Ahmed Fouad MD ${ }^{b}$, Magdy BedirAli ${ }^{a}$, \\ Saeed Abo El-Ez El-Baz ${ }^{a}$ \\ ${ }^{\text {[a] }}$ Professor, Otorhinolaryngology Head and Neck Surgery Department, Faculty of Medicine, Zagazig \\ University, Egypt. \\ ${ }^{[b]}$ Lecturer, Otorhinolaryngology Head and Neck Surgery Department, Faculty of Medicine, Zagazig \\ University, Egypt. \\ ${ }^{[c]}$ Resident, Otorhinolaryngology Head and Neck Surgery Department, Faculty of Medicine, Zagazig \\ University, Egypt.
}

\begin{abstract}
Background: Fat myringoplasty had been described over 50 years to be a safe and simple option for myringoplasty. Platelet rich plasma [PRP] is a simple and minimally invasive method to obtain a high concentrate of autologous growth factors that can facilitate wound healing.

Objectives: To assess the effectiveness of using fat and PRP in myringoplasty.

Patients and methods: Twenty patients with small to medium sized tympanic membrane perforations were enrolled in this study. Under local anesthesia, the margin of the perforation was freshened, then a single piece of ear lobule fat graft, approximately double the size of the perforation, was placed through the perforation, then the PRP was applied over the fat graft.

Results: Successful TM perforation repair was achieved in 36 ears [85.7\%]. 100\% success rate was obtained in small peroration and 79.3\% in medium sized perforation. There was significant postoperative improvement in the mean air bone gap [ $p=0.0016]$.

Conclusion: Adding PRP to fat myringoplasty is a safe, effective, cost saving and minimally invasive procedure. It is suitable to repair small as well as moderate sized TM perforations.

Keywords: Myringoplasty, platelet rich plasma, tympanic membrane. Ear lobule fat

Corresponding author; Ahmed Roshdy Ebrahim.

Address: Otorhinolaryngology, Head and Neck Surgery Department, Faculty of Medicine, Zagazig University, Egypt.

Tel: +201061166410 .

Email: ahmedroshdyemail@gmail.com
\end{abstract}

\section{INTRODUCTION}

T he physiologic function of the tympanic membrane (TM) is not only transmission of sound waves to the middle ear sound conducting system, also it has an important role in middle ear protection against external environment and also against nasopharyngeal reflux "middle ear cushion" [1]. This can explain why unrepaired TM perforations can significantly impair the quality of life ${ }^{[2]}$.

Many materials are available for closure of TM perforations such as perichondrium ${ }^{[3]}$, temporalis fascia ${ }^{[4]}$ vein graft ${ }^{[5]}$, and cartilage ${ }^{[6]}$.

Fat graft myringoplasty has been first described in 1962 by Ringenberg as a simple technique for repairing of TM perforations ${ }^{[7]}$. Closure rates have been reported varying $76 \%$ to $92 \%$ of cases in small TM perforation [ ${ }^{8,9}$ ].

Platelet rich plasma [PRP] is a simple and minimally invasive method to obtain a high concentrate of autologous growth factors ${ }^{[10]}$. In their clinical study of PRP application during repair of large TM perforation, ElAnwar et al ${ }^{[11]}$ proved a postive PRP effect on healing without reported complication.

The aim of this study was to assess the effectiveness of adding PRP in case of fat myringoplasty under local anesthesia to enhance healing.

This prospective study was conducted on 42 patients who had small to medium sized dry central TM perforation. TM perforation was considered small sized if its surface area is less than quarter of TM surface, and considered medium sized if its surface area is more than quarter of the TM surface and less than half of the TM surface, as described by Saliba ${ }^{[12]}$.

The TM perforation must be dry with apparently healthy middle ear mucosa for one month at least before surgery. Patients with TM perforations larger than one half of TM size were excluded from the study. Patients with active ear discharge, cholesteatoma, marginal and attic perforations and those with 
suspected ossicular pathology, air bone gap more than $35 \mathrm{~dB}$, were also excluded.

A written formal consent to participate in the study was signed by enrolled subjects and the study was approved by the Institutional Review Board [IRB].

All patients were subjected to full history taking, general and local examination, including otoscopic and microscopic ear examination with audio logical evaluation in the form of pure tone audiometry at the speech frequencies 500, 1000, $2000 \mathrm{~Hz}$ and the average air bone gap [ABG] in those frequencies was recorded. In addition, preoperative laboratory tests including CBC, ESR and coagulation profile were done to look for any contraindications to PRP use.

\section{Local anesthesia:}

In the preoperative preparation room, topical anesthesia was achieved by insertion of a pack soaked with lidocaine [2.5] + prilocaine [2.5\%] cream pack [Manufactured by Astra Zeneca] in the external auditory canal, then infiltration of skin of the posterior surface of the ear lobule was performed by $2 \%$ lidocaine.

\section{Preparation of the autologous PRP}

After local anesthesia, $5 \mathrm{ml}$ of peripheral venous blood was drawn from the patient with a 16 or 18 gauge butterfly needle or syringe to avoid irritation and damage of the platelets. Extracted blood was immediately placed in a plain vacuum tube [without anticoagulant or calcium], $5 \mathrm{~mL}$ in each tube, then immediately centrifuged using a tabletop centrifuge [Zhengji 800-centrifuge, china] machine for 12 minutes at $3200 \mathrm{rpm}$. This causes the blood to be separated out into the three layers formed because of their density; the bottom layer containing red blood cells; the middle layer containing PRP [about 1.5 $\mathrm{ml}^{3}$ ] consisting of platelets and white blood cells [WBCs; buffy coat]; and the top layer containing platelet-poor plasma.. The PRP layer was taken out later at time of use. This method for PRP prepation was previously described by El-Anwar et al ${ }^{11,13}$

\section{Surgical procedure}

After withdrawal of the needed venous blood, the patients were transferred to the operating room and positioned in a supine position with their face turned to the opposite side of the perforation. The surgical field is scrubbed and draped as usual for otologic surgery, then under microscope the EAC pack was removed and the EAC was washed with saline. Then 2 small pieces of cotton soaked in a mixture of $1 / 1000$ adrenaline and $2 \%$ lidocaine were inserted medial and lateral to the TM perforation. Then a transverse incision, nearly $1 \mathrm{~cm}$ length, was made in the posterior surface of ear lobule to harvest a single piece of ear lobule fat of suitable size using Allis tissue forceps.

Thereafter, the previously applied cotton pieces were removed and trimming of the perforation margin was performed [Figure 1 A]. At this time, the harvested fat graft was inserted through the TM perforation and adjusted making most of the fat piece lying medial and small part lateral to the perforation, resembling the shape of the top of an ice berg [Figure 1B], not resembling the shape of an hourglass as previously described in literature ${ }^{[8,9]}$.

The prepared autologous PRP was inserted [as a plug] into external auditory canal [EAC] on the lateral surface of the graft and TM remnant [Figure 1C]. Large pieces of gel foam were inserted filling the medial part of EAC to support the PRP; meatal pack soaked in gentamycin ointment was placed for one week, the incision of the ear lobule was sutured using 3/0 silk suture. No ear dressing was used and patients were discharged after the procedure with postoperative instruction.

\section{Postoperative follow up}

Pack and stitches was removed after one week and the patients were followed up at weekly intervals for one month and monthly for 3 months, Graft was considered as successfully taken if there was no residual perforation after 3 months.

Audio logic assessment was performed after 3 months in the form of pure tone audiometry at the speech frequencies 500, $1000,2000 \mathrm{~Hz}$ and the average ABG in those frequencies was recorded.

\section{RESULTS}

42 patients; 24 females (57\%) and 18 males (43\%) who had dry central TM perforation caused by tubo-tympanic chronic suppurative otitis media; The size of TM 
perforation was small in 13 cases $(30.95 \%)$ and medium in 29 cases $(69.05 \%)$. The perforation was in right TM in 23 patients $(55 \%)$ and in left TM in 19 patients $(45 \%)$. The age of the patients ranged from 12 to 42 years old with a mean of (26.5) years.
Successful TM perforation closure was achieved in 36 ears $(85.71 \%)$. Success rate in small TM perforation was $100 \%$ and in medium sized perforation was $79.3 \%$. [Table 1]. None of the patients developed worsening of hearing, tinnitus, vertigo, bleeding, taste disturbance or hyperkeratosis.

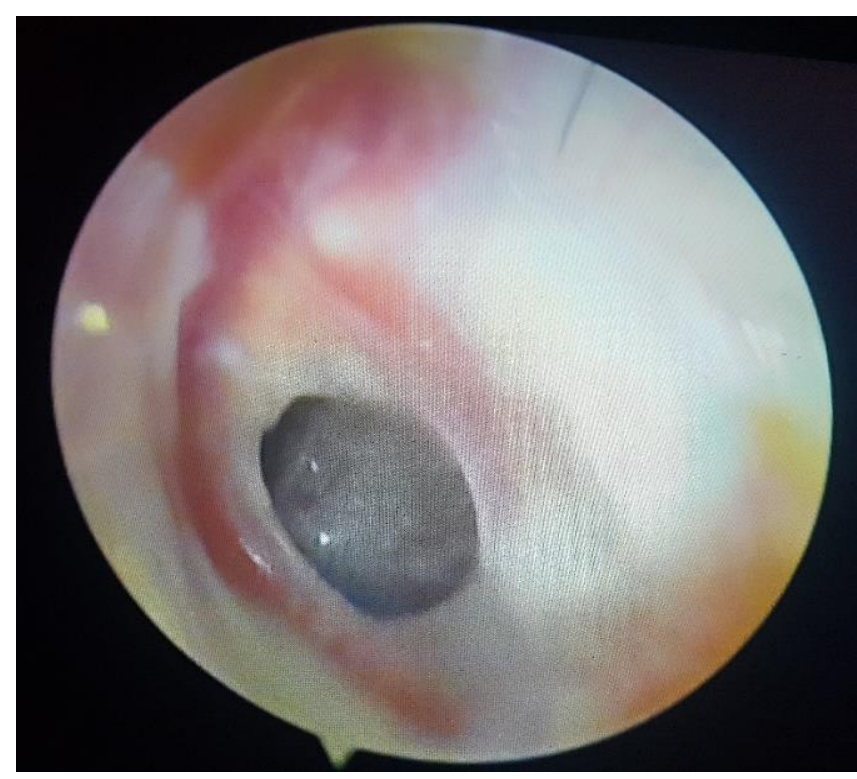

Fig. (9): showing pre-operative left ear central perforation

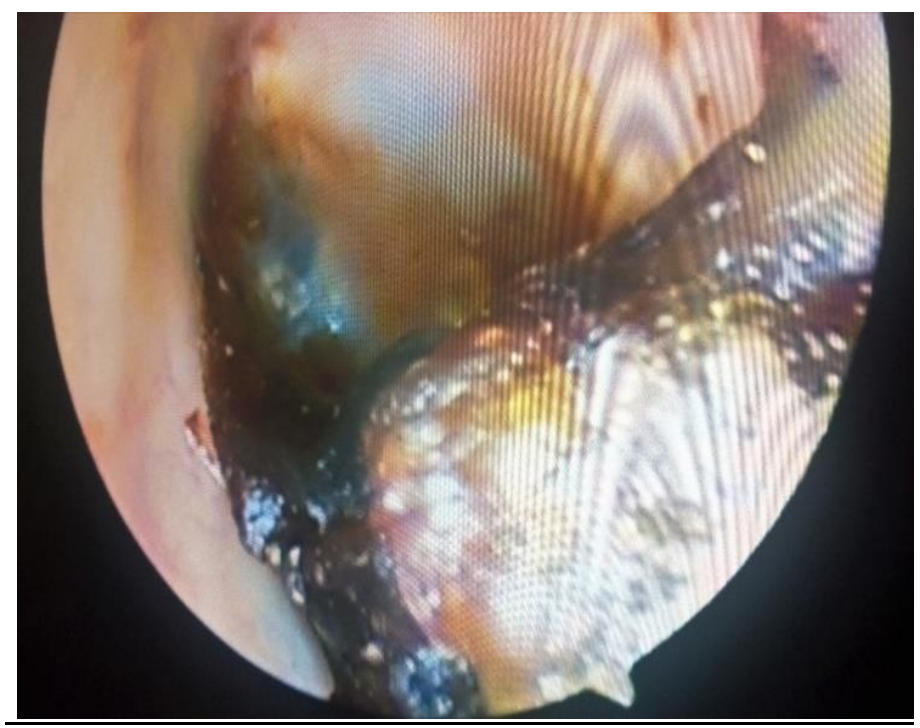

Fig. (10): showing the PRP graft after two days of operation. 


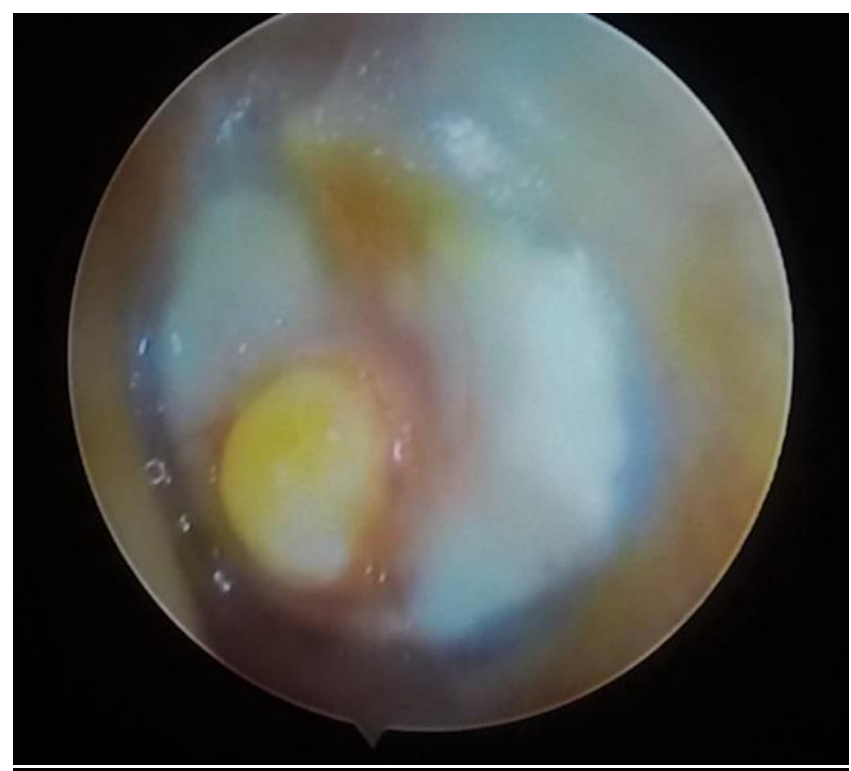

Fig. (11): showing the fat graft in place with healing two weeks' post-operative

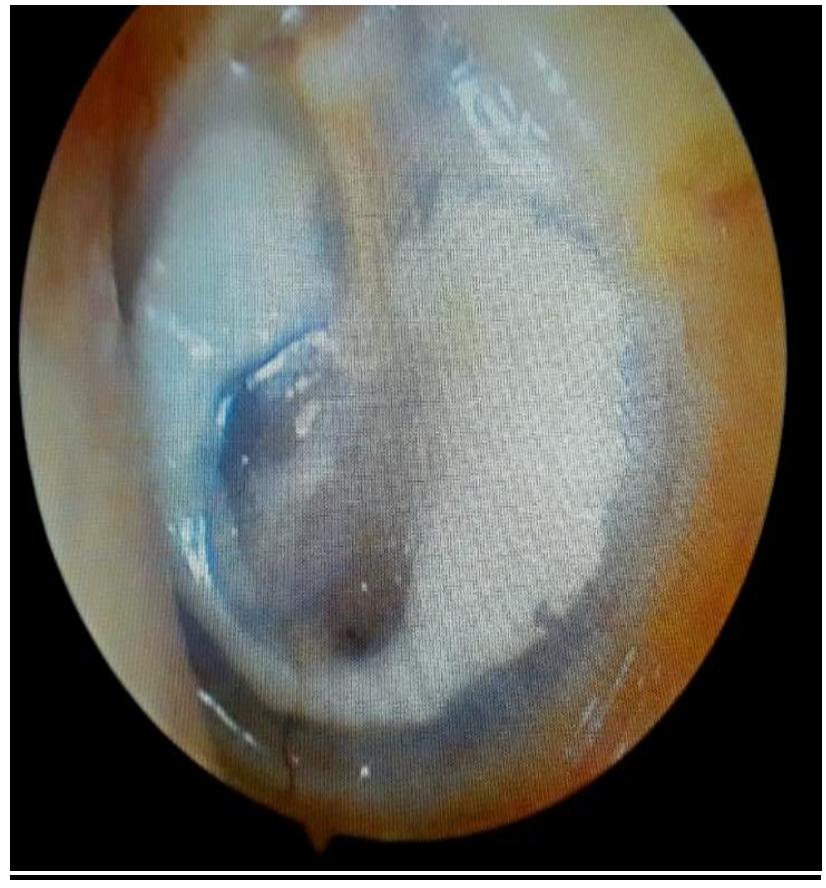

Fig (12) showing healing with fat graft absorption 6ms. postoperative. 
Table 1: Incidence of successful cases, closure rate, among small and medium sized TM perforations. NS: non-significant.

\begin{tabular}{|c|c|c|c|c|c|c|c|}
\hline & & & & & & Fisher's Exact test & p-value \\
\hline & & & Perfor & ion group & & & \\
\hline & & & Small sized & Medium sized & Total & & \\
\hline Result & success & Count & 13 & 23 & 36 & 3.138 & $\begin{array}{l}\mathbf{0 . 1 5 3} \\
\text { [NS] }\end{array}$ \\
\hline & & \% within size & & & & & \\
\hline & & & $100.0 \%$ & $79.3 \%$ & $85.7 \%$ & & \\
\hline & failure & Count & & & & & \\
\hline & & & $\mathbf{0}$ & 6 & 6 & & \\
\hline & & \% within size & & & & & \\
\hline & & & $0.0 \%$ & $20.7 \%$ & $14.3 \%$ & & \\
\hline & Total & Count & & & & & \\
\hline & & & 13 & 29 & 42 & & \\
\hline & & \% within size & & & & & \\
\hline & & & $100.0 \%$ & $100.0 \%$ & $100.0 \%$ & & \\
\hline
\end{tabular}

The mean preoperative air bone gap at speech frequencies was $17.7 \pm 4.2 \mathrm{~dB}$ while the mean postoperative air bone gap was $13.5 \pm 3.6$ with significant improvement $[\mathrm{P}=0.0016]$ [table 2]

Table 2: pre and postoperative air bone gap results. S: significant

\begin{tabular}{|c|c|c|c|c|}
\hline & $\begin{array}{c}\text { Air bone } \\
\text { gaps }\end{array}$ & \multirow{2}{*}{$\begin{array}{c}\text { T. } \\
\text { value }\end{array}$} & \multirow{2}{*}{$\begin{array}{c}\text { p. } \\
\text { value }\end{array}$} & \multirow[t]{2}{*}{ Significance } \\
\hline & Mean \pm SD & & & \\
\hline Pre-operative & $17.7 \pm 4.2$ & $t=$ & \multirow{2}{*}{0.0016} & \multirow{2}{*}{$\mathbf{S}$} \\
\hline $6 \mathrm{~ms}$ post-operative & $13.5 \pm 3.6$ & 3.3955 & & \\
\hline
\end{tabular}

The mean duration of the procedures was $24 \pm 3.5$ minutes including duration of PRP preparation and scrubbing. The mean duration of the actual operative procedures, starting by the skin incision, was $12.8 \pm 2.5$ minutes.

None of the patients developed worsening of hearing, tinnitus, vertigo, bleeding, taste disturbance or hyperkeratosis. One patient with history of previously treated otomycosis developed reappearance of fungal infection and failure of occlusion of the TM perforation.

All patients tolerated the procedure under topical anesthesia with no need to use an oral anxiolytic prior to the procedure, or conscious sedation measures for the procedure itself. Postoperative pain was tolerable in all patients with no need for injectable analgesic.

\section{DISCUSSION}

Trans tympanic myringoplasty has many advantages over the classic myringoplasty with elevation of tympanomeatal flap. It is simpler, more direct, and can be easily performed under local anesthesia. Additionally, no elevation of the tympanomeatal flap resulting in less risk of injury of the tympanic annulus, chorda tympani, and middle ear ossicles; also less risk of medialization or lateralization of the TM. However, in this approach, exploration of the tympanic cavity could not be performed and the integrity and mobility of the ossicular chain could not be properly tested.

Fat graft myringoplasty is usually performed by trans tympanic approach under local anesthesia. It has the same advantages of trans tympanic myringoplasty, in addition, the 
donor site in fat myringoplasty is easy accessible through a small skin incision.

Fat graft myringoplasty is originally described for small TM perforation with reported success rate variable from $76 \%$ to $92 \%$ of cases ${ }^{[8,9,14-17]}$. Deddens et al. ${ }^{[17]}$ considered TM perforation size as a crucial factor for a successful fat myringoplasty, he used fat graft myringoplasty for perforation size ranging between $5 \%$ to $30 \%$ of the TM surface.

On their study of fat myringoplasty in different TM perforations sizes; Konstantinidis et al ${ }^{[18]}$ found that the success rate was $100 \%$ in the group of perforations sized $0 \%$ to $10 \%$ of TM, $94.45 \%$ in the Group of $11 \%$ to $20 \%, 93.75 \%$ in the Group $21 \%$ to $30 \%$, and $60 \%$ in the Group $41 \%$ to $50 \%$.

The worse results of fat myringoplasty in perforations larger than $30 \%$ lead to thinking in an additive substance that enhance the results even in larger perforations. Saliba et al [12] added hyaluronic acid disc to support the fat plug leading to marked improvement of the results as they reported success rate of $75 \%, 78 \%, 83 \%$, and $100 \%$ in total, large, medium, and small sized perforation. However, we found that hyaluronic acid disc, as a synthetic material, is not always available and costly.

In the current study, we tried to use PRP to enhance healing and to support the fat graft in similar idea of addition of hyaluronic acid, however PRP is an autogenous material, not artificial, and more economic than hyaluronic acid. In the current study, addition of PRP to fat myringoplasty resulted in $100 \%$ closure rate in small TM perforation and $79.3 \%$ in medium sized TM perforation.

In the first human study on use of PRP in myringoplasty, El-Anwar et al [11] documented its safety anf effecacy in improving success rate. But up till now no study on the PRP use with fat myringoplasty. PRP is growth factors carrier so it enhances endothelial, epithelial, and epidermal regeneration, stimulates angiogenesis, enhances collagen synthesis and soft tissue healing, decreases scarring, and promotes the hemostatic response to injury ${ }^{[19]}$. The presence of WBCs in high concentrations in our chosen technique for obtaining PRP has a significant role against infection with its bactericidal effect ${ }^{[20,21]}$. This was evident in our study as we did not encounter any case of suppurative infection.

Our study was conducted on adult population with perforation sized less than $50 \%$ in the absence of other middle ear pathologies with follow up period less than 12 months; the closure rate in our study was $85.7 \%$. By comparing our results to the meta analysis that was conducted by Tan et al., ${ }^{[22]}$ on type 1 tympano-plasty performed in 214 studies involving 26,097 patients, we found that; the overall closure rate in this metaanalysis was $86.6 \%$, the closure rate in the studies conducted on TM perforations sized less than $50 \%$ was $85.56 \%$ [74 studies, 5859 patients], the closure rate in the studies that were conducted with follow up periods less than 1 year was $85.61 \%$ [33 studies, 8279 patients], the closure rate in the studies on adult populations was $89.25 \%$ [26 studies, 2049 patients]. the closure rate in the studies used fat as a grafting material was $86.52 \%$ (22 studies 1.507 patients) ${ }^{[22]}$.

Thus, Myringoplasty using fat and PRP is a safe, minimally invasive, and effective procedure that is suitable for repairing small and medium sized TM perforations. However, further studies with larger sample and studies comparing this technique with simple fat myringoplasty and with fat and hyaluronic acid myringoplasty are needed.

\section{CONCLUSION}

Adding PRP to fat myringoplasty is a safe, effective, cost saving and minimally invasive procedure. It is suitable to repair small as well as moderate sized TM perforations.

\section{REFERENCES}

1. Boedts, M. Paper patching of the tympanic membrane as a symptomatic treatment for patulous eustachian tube syndrome. The Journal of Laryngology \& Otology., 2014, 128[03], pp.228-235.

2. Sheahan, P., O'Dwyer, T. and Blayney, A. Results of type 1 tympanoplasty in children and parental perceptions of outcome of surgery. The Journal of Laryngology \& Otology., 2002, 116[06], pp.430-434.

3. Quraishi MS, Jones NS. Day case myringoplasty using tragal 
perichondrium. Clinical Otolaryngology \& Allied Sciences 1995; 20[1]: 12-14.

4. Storrs L. Myringoplasty with the use of Fascia Grafts. Arch Otolaryngol 1961; 74:45-49.

5. Tabb HG. Closure of perforations of the tympanic membrane by vein grafts: a preliminary report of 20 cases. Laryngoscope 1960; 70:271-286.

6. Klacansky J. Cartilage myringoplasty. The Laryngoscope 2009; 119[11]: 2175-2177.

7. Ringenberg JC. Fat graft tympanoplasty. The Laryngoscope 1962; 72[2]: 188-192.

8. Terry RM, Bellini MJ, Clayton MI, Gandhi AG: Fat graft myringoplasty: a prospective trial. Clin Otolaryngol Allied Sci 1998; 13: 227-229.

9. Mitchell RB, Pereira KD, Lazar RH. Fat graft myringoplasty in children-a safe and successful day-stay procedure. The Journal of Laryngology \& Otology 1997: 111[02]: 106108.

10.Creaney L, Hamilton B. Growth factor delivery methods in the management of sports injuries: the state of play. Br J Sports Med 2008; 42[5]: 314-320.

11.El-Anwar MW, Sayed El-Ahl MA, Zidan AA, Mohammad Yacoup AA. Topical use of autologous platelet rich plasma in myringoplasty. Auris Nasus Larynx 2015; 42: 365-368.

12.Saliba I. Hyaluronic acid fat graft myringoplasty: how we do it. Journal compilation Clinical Otolaryngology 2008; 33: 607-628.

13.El-Anwar MW, Nofal AA, Khalifa M, Quriba AS. Use of autologous platelet-rich plasma in complete cleft palate repair. Laryngoscope 2016; 126[7]: 1524-8.

14. Ayache S, Braccini F, Facon F, Thomassin JM: Adipose graft: An original option in myringoplasty. Otol Neurotol 2005; 26: 554555.

15.Gross CW, Bassila M, Lazar RH, Long TE, Stagner S. Adipose plug myringoplasty: An alternative to formal myringoplasty techniques in children. Otolaryngol Head Neck Surg 1989; 101:617-20.

16.Dursun E, Dogru S, Gungor A, Cincik H, Poyrazoglu E, Ozdemir T. Comparison of paper-patch, fat, and perichondrium myringoplasty in repair of small tympanic membrane perforations. Otolaryngology--Head and Neck Surgery 2008; 138[3]: 353-356.

17.Deddens AE, Muntz HR, Lusk RP. Adipose myringoplasty in children. Laryngoscope 1993; 103:216-9.
18. Konstantinidis I, Malliari H, Tsakiropoulou E, Constantinidis J. Fat myringoplasty outcome analysis with otoendoscopy: who is the suitable patient? Otology \& Neurotology 2013; 34[1]: 95-99.

19.Smith RG, Gassmann CJ, Campbell MS. Platelet-rich plasma: properties and clinical applications. The Journal of Lancaster General Hospital 2007; 2 [2]: 73-77.

20.Tate KS, Crane DM. Platelet rich plasma grafts in musculoskeletal medicine. Journal of Prolotherapy 2010; 2[2]: 371-376.

21.Bielecki TM, Gazdzik TS, Arendt J, Szczepanski T, Król W, Wielkoszynski T. Antibacterial effect of autologous platelet gel enriched with growth factors and other active substances; an in vitro study. The Journal of Bone and Joint Surgery 2007; 89 [3]: 417-420.

22. Tan, H.E., Santa Maria, P.L., Eikelboom, R.H., Anandacoomaraswamy, K.S. and Atlas, M.D. Type I Tympanoplasty Meta-Analysis: A Single Variable Analysis. Otology \& neurotology: official publication of the American Otological Society, American Neurotology Society [and] European Academy of Otology and Neurotology, 2016. 